\title{
Compound stimulus transfer among forward and backward conditioning situations ${ }^{1}$
}

\author{
WILLIAM W. GRINGS and ARTHUR \\ ZEINER ${ }^{2}$ University of Southern \\ California, University Park, Los Angeles, \\ Calif. 90007
}

Sixty Ss were assigned to five independent groups of a three-stage experiment. In Stage 1, four groups received forward conditioning, and a fifth was an unpaired control. In Stage 2, a new $C S$ received forward conditioning, backward conditioning, or unpaired presentations. In Stage 3, responses to components and compounds made up of CSs from Stages 1 and 2 were tested. Groups did not differ on a pretest. All forward groups conditioned in Stage I. In Stage 2, forward and unpaired groups differed, but backward groups did not differ from either forward or unpaired groups. In Stage 3, the forward-forward group differed significantly from the unpaired group. Backward groups did not differ from the forward group but differed at a marginally significant level from the unpaired group.

An investigation was reported recently in which counterconditioning of fear and relaxation responses was demonstrated, using the galvanic skin response and digital pulse volume as the response measures (Grings \& Uno, 1968). To provide an arrangement for demonstrating response interaction, the compound stimulus-transfer paradigm was used (Grings \& O'Donnell, 1956). Ss were first conditioned to respond differentially to two colored light stimuli by associating one of the stimuli with an electric shock and not reinforcing the other stimulus. This was assumed to condition the response of fear to the reinforced colored light. Then the Ss were conditioned to relax voluntarily when a visual stimulus word was projected (as contrasted to no response to a control word). This procedure employed relaxation techniques modified from those of Jacobson (1938). After training effects could be demonstrated to both responses, two stimuli (one eliciting fear and the other eliciting relaxation) were presented as a stimulus compound, and response magnitudes were compared for this compound stimulus and the individual stimuli.

The previous study presented difficulties in the conditioning of the relaxation response. For one thing, it did not follow conventional conditioning procedures in the sense that no unconditioned response was involved. The $S$ was trained merely to perform a voluntary response upon appearance of the signal. Other means were then sought for eliciting, by direct stimulation, a response that might be labeled as relaxation or relief and which might be assumed to be antagonistic to fear. Barlow (1956) had presented data supporting the hypothesis that stimuli associated with the offset of shock acquire positive properties as contrasted to stimuli associated with shock onset that acquire negative properties. Association of a stimulus with shock offset is provided by the circumstances of backward conditioning.

Reasoning from the above possibilities, the present experiment was designed to answer two specific questions. First, would forward-as compared to backward-conditioning operations lead to aversive as compared to positive properties as hypothesized by Barlow? Second, would stimulus compounds made up of differently treated CSs show the effects of these differential treatments? A GSR conditioning procedure consisting of three stages was used: The first stage presented forward-conditioning trials to one stimulus, the second stage presented to different subgroups either backward-conditioning or forward-conditioning experience to a second stimulus, and, finally, the third phase tested response to combinations of the stimuli.

Sixty undergraduate students from introductory psychology classes served as Ss as part of a class requirement. The students were assigned by a table of random numbers to five groups of $12 \mathrm{Ss}$ each. During Stage 1, forward conditioning of the GSR to visual stimuli (colored light dots), with shock UCS and a .5-sec CS-UCS interval, was carried out for four of the groups. The remaining group served as an unpaired (random) control group. Stage 2 started with a test trial on a second light CS for all Ss. Then two of the original forward-conditioned groups and the original unpaired group received backward-conditioning training on the new CS. One of the previously forward-conditioned groups received a 4.0-sec UCS-CS interval and the other two groups received a $1.5-\mathrm{sec}$ UCS-CS interval. One of the original forward-conditioned groups received further forward conditioning on the second CS, while the fifth group was given unpaired CS and UCS presentation. In Stage 3, transfer effects were tested by trials on both CSs individually and in pairs. CS duration and UCS duration were $.5 \mathrm{sec}$ for all Ss. The intertrial interval was varied randomly in 10-sec steps from 20 to $50 \mathrm{sec}$.

The general procedure consisted of having the Ss set the initial shock level at a point of subjective discomfort. Then two adaptation trials were given on each CS with trial order counterbalanced. In both Stages 1 and 2 , there were 12 acquisition trials. Of these 12 acquisition trials, 4 were nonreinforced and used as test trials. Stage 3 was a continuation of Stage 2, with the addition of 2 compound test trials and 4 component test trials interspersed in a systematic-order rotation.

The GSR was recorded as a dc resistance change from the second and third fingers of the right hand. Silver electrodes, $1 / 2 \times 5 / 8$ in., were taped to the fingertips and led to a modified Darrow bridge, the output of which was recorded on an Offner Type $\mathrm{R}$ dynagraph. Conditioned stimuli were produced by a Grason-Stadler multiple-stimulus projector. The UCS was a dc shock produced by a Grass Model S-5 stimulator and delivered by 11/16-in.-diam silver electrodes placed 2 in. apart on the volar surface of S's left arm. Stimulus durations and interstimulus intervals were controlled by Hunter timers, intertrial intervals by a Gerbrands stimulus programmer.

\section{RESULTS}

All responses with a latency of 1 to $5 \mathrm{sec}$ after CS onsets were read from response onset to peak. This value was transformed to the unit square root of conductance change. Responses on adaptation trials were used to determine equivalence of initial groups. There were no significant differences in responding between groups or between the different CSs during the adaptation series.

The average response on all four test trials during the acquisition phase of Stage 1 was obtained. Each of the forward-conditioning groups differed significantly from the control group in Stage 1 (p, in all cases, less than .05). They did not differ significantly from each other.

At the start of Stage 2, Ss responded to the new CS much as they had responded to the CS at the end of Stage 1. That is, the experimental groups gave significantly larger responses to the new stimulus than the control group did. Again, the responses of the various groups for all four test trials in the second stage were averaged. The group receiving continued forward conditioning performed highest throughout Stage 2. In the case of both backward-conditioning groups, the response to the second CS decreased with 
Table 1

Transfer from Components to Compounds Involving Forward and Backward Conditioning of Components $(N=12$ in Each Group)

\begin{tabular}{llccc}
\hline \multicolumn{2}{c}{ Treatment Group } & $\begin{array}{c}\text { Compound } \\
\text { Test One }\end{array}$ & $\begin{array}{c}\text { Compound } \\
\text { Test Two }\end{array}$ & $\begin{array}{c}\text { Average } \\
\text { CS1 }\end{array}$ \\
\hline Forward & CS & 1.71 & 1.47 & 1.59 \\
Forward & Forward & 1.35 & 1.25 & 1.30 \\
Forward & Backward 1.5 sec & 1.67 & 1.16 & 1.42 \\
Forward & Backward 4.0 sec & 1.24 & .90 & 1.07 \\
Random & Random & 1.38 & .75 & 1.07 \\
\hline
\end{tabular}

continued paired-stimulus presentation. unpaired with shock. It is not possible to Overall, the backward-training groups did tell whether this represented adaptation or not differ from the unpaired group in extinction of generalized responding to the Stage 2. second stimulus as contrasted to the

The responses to the compound stimuli development of a new response. in Stage 3 are given in Table 1 . Significant Consequently, the transfer effects that compound stimulus transfer effects were were demonstrated are more analogous to found between groups. The compound of those obtained by tests of simple stimulus two forward-conditioning components was compounds composed of components, one superior to and significantly different from of which had previously been reinforced the unpaired group $(t=2.22 ; \mathrm{df}=22)$. The and the other of which had not been group receiving the combination of the reinforced. In other words, the differences forward cue and the 1.5 -sec backward cue in the transfer test trials on paired stimuli differed from the unpaired group at a are of the same order of magnitude as was marginally significant level $(t=1.96)$. A obtained in studies where component similar difference was found for the 4.0-sec stimuli were either reinforced or backward compound $(\mathrm{t}=1.87)$.

$$
\text { DISCUSSION }
$$

nonreinforced and then tested in combinations or pairs (Grings \& O'Donnell,

The results obtained in Stage 2 above do not support the assumption that backward conditioning had been obtained. Response to the second stimulus decreased consistently throughout Stage 2 to a point where, by the end of the stage, the backward-conditioning groups were responding no more to the second stimulu than was the group receiving that stimulus active-passive, and safe-dangerous. While there were changes in the responses from before to after the experiment, the changes were not different for the various groups. A subsequent study exploring further the role of 'Ss' conceptualizations of the backward-conditioning arrangement has been reported recently (Zeiner \& Grings, 1968).

\section{REFERENCES}

BARLOW, J. A. Secondary motivation through classical conditioning: A reconsideration of the nature of backward conditioning. Psychological Review, 1956, 63, 406-408.

EVANS, W. O. Reducing either positive or negative tendencies to a new stimulus associated with shock. Joumal of the Experimental Analysis of Behavior, 1962, 5 , 335-337.

GRINGS, W. W., \& O'DONNELL, D. E. Magnitude of response to compounds of discriminated stimuli. Joumal of Experimental Psychology, 1956, 52, 354-358.

GRINGS, W. W.. \& UNO, T. Counterconditioning: $\mathrm{Fear}$ and relaxation. Psychophysiology, 1968, 4, 479-485.

GRINGS, W. W., UNO, T., \& FIEBIGER, J. Component to compound stimulus transfer. Psychonomic Science, 1965, 3, 63-64.

JACOBSON, E. Progressive relaxation. Chicago: University of Chicago Press, 1938.

WAGMAN, W., \& ALLEN, J. D. The development of a conditioned positive reinforcer based upon the termination of shock. Psychonomic Science, 1964, 1, 363-364.

ZEINER, A., \& GRINGS, W. W. Backward conditioning: A replication with emphasis on conceptualizations by the subject. Journal of Experimental Psychology, 1968, 76, 232-235. NOTES

1. This research was supported in part by Grant MH-3916 from the National Institute of Mental Health

2. Now at the University of California Medical Center, San Francisco. 